\title{
Measuring Cluster Relaxedness
}

\author{
Blythe Moreland \\ University of Michigan
}

Office of Science, Science Undergraduate Laboratory Internship Program (SULI)

SLAC National Accelerator Laboratory

Menlo Park, California

\begin{abstract}
August 18, 2011
Prepared in partial fulfillment of the requirements of the Office of Science, Department of Energy's Science Undergraduate Laboratory Internship, under the direction of Risa Wechsler at the Kavli Institute for Particle Astrophysics and Cosmology at SLAC.
\end{abstract}

Participant: $\quad$ Blythe Moreland

Research Advisor: Risa Wechsler 


\section{Contents}

1 Introduction $\quad 2$

2 Methods $\quad 3$

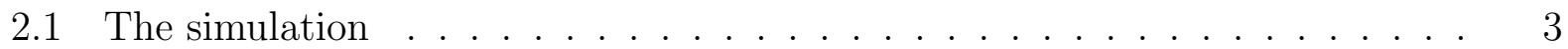

2.2 The measurements . . . . . . . . . . . . . . . . . . . . . 4

3 Results and discussion $\quad 6$

3.1 Relaxedness measures and halo properties . . . . . . . . . . . . 7

3.2 Relaxedness and major mergers . . . . . . . . . . . . . . . . . 11

4 Conclusion and future work $r$

$\begin{array}{llr}5 & \text { Acknowledgements } & 18\end{array}$ 


\section{Introduction}

When is a dark matter halo "relaxed"? In our efforts to understand the structure of the universe, dark matter simulations have provided essential grounds for theoretical predictions. These simulations provide a wealth of ways of parameterizing and measuring the features of astronomical objects. It is these measurements on which we base comparisons of our world and our attempts to re-create it. One of the essential questions dark matter simulations help address is how dark matter halos evolve. How does one characterize different states of that evolution? The focus of this project is identifying cluster relaxedness and how it relates to the internal structure of the halo.

A dark matter simulation consists of an $N$-body simulation which takes an initial set of positions and velocities of the dark matter particles and evolves them under the influence of gravity [6]. Though scientists have so far not been able to detect dark matter particles, the information from these simulations is still valuable especially given the relationship between dark matter halos and galaxy clusters. Galaxies sit within dark matter halos and recent evidence points to filaments of dark matter forming the framework on which galaxy clusters grow [7]. A dark matter halo is a collapsed group of gravitationally bound dark matter particles. Subsets of bound particles form subhalos or substructures. The dark matter simulation is carried out over time - with decreasing redshift $(z)$ or increasing scale factor $\left(a=\frac{1}{1+z}\right)$. (Thus, $z=0$ or $a=1.0$ is present-day.) The merger history of a halo can be represented pictorally by a merger tree. A major merger event occurs when a structure joins the main halo with the mass ratio between it and the main halo being above a certain threshold. These events mark important points in the halo's evolution. And it is at these events that one hopes, and perhaps is more likely, to relate measures of relaxedness to this mass accretion.

Cluster relaxedness is not a well-defined concept. Rather a set of qualities are defined that one expects a "relaxed" cluster to have. One expects a relaxed halo to have a roughly 
isotropic density distribution. Most of the particles should be part of the main halo rather than bound in substructures. Taking into account kinematic information of the halo, one does not expect a proportion of the particles' energy in kinetic energy that goes far beyond virial equilibrium.

With our measures of cluster relaxedness, we want to investigate its relationship to these major merger events. We'll first look at how measurements of different aspects of relaxedness relate to each other and to other aspects of a halo's internal structure. We'll then look at how these measurements behave in response to major mergers.

\section{Methods}

\subsection{The simulation}

Our dark matter halos are from the Rhapsody (Re-simulated Halo Sample for Statistical Observable-mass Distribution Study) catalog [9]. The sample includes 110 halos with virial mass $\log _{10} M_{v i r}=14.8 \pm 0.05 \mathrm{M}_{\odot} / h$ and this puts the halos in the regime of "massive" halos. The parent simulation is called Carmen of the LasDamas suite of simulations that originally ran with a $1000 \mathrm{Mpc} / \mathrm{h}$ size box and $1120^{3}$ collisionless particles and a softening scale of $25 \mathrm{kpc} / \mathrm{h}$. Wu's process of curating these massive halos was to select halos in a high but narrow mass bin, zoom-in on them and re-simulate them at higher resolution. Such a bin omits enough particles to be able to run for high resolution but admits a statisticially appreciable number of halos. The resulting smoothing scale is $6.7 \mathrm{kpc} / \mathrm{h}$. This resolution gives us significant substructures and a rich, unique probe into halo evolution. The data cited

in the ensuing sections is the output of the halo-finder and merger tree at 100 "snapshots" of the simulation. These snapshots are spaced equally in $\log a$, thus the time in gigayears (Gyr) between each snapshot is not constant. 


\subsection{The measurements}

The relaxedness measurements (which will be shortened to RMs) considered here are taken from the literature and in particular from Neto's paper on CDM halo structure [4]. They are described below:

\section{(i) Concentration:}

Navarro, Frenk \& White [3] proposed a simple model to approximate the density profile of a dark matter with two free parameters:

$$
\frac{\rho(r)}{\rho_{\text {crit }}}=\frac{\delta_{c}}{\left(r / r_{s}\right)\left(1+r / r_{s}\right)^{2}}
$$

$\rho_{\text {crit }}$ is the critical density of the universe - the density required for a closed universe. $\delta_{c}$ is called the characteristic density and $r_{s}$, the scale radius. This profile has done well at parameterizing dark matter halos close to virial equilibrium. The NFW profile indicates two major regimes where different power laws dominate. This $r_{s}$ tells us something about the shape of the density profile as it corresponds to the transition between the two power laws. The concentration measurement is defined as $c_{v i r}=r_{v i r} / r_{s}$ where $r_{v i r}$ is the virial radius of the halo. This value relates to how much of the halo is in this denser region.

(ii) NFW fit error :

Since the NFW profile best describes large, relaxed halos. The $\chi^{2}$ value [4] used to evaluate the goodness of fit when fitting the profile may be an indicator of relaxedness:

$$
\chi^{2}=\frac{1}{N_{\text {bins }}-1} \sum_{i=1}^{N_{\text {bins }}} \frac{1}{w_{i}}\left[\log _{10} \rho_{i}-\log _{10} \rho_{N F W}\left(\delta_{c} ; r_{s}\right)\right]^{2}
$$

Where $w_{i}$ is the number of particles in bin $i$.

For (i) and (ii) the fitting process plays a role in determining the values. The IDL function MPFIT.pro was used to minimize the $\chi^{2}$ value. The halo is binned initially in 32 bins spaced equally in $\log _{10}\left(r / r_{v i r}\right)$, with the innermost bin beginning when $\log _{1} 0\left(r / r_{v i r}\right)=-2.5$ 
and the outermost bin ending at $r_{v i r}$. Then bin edges were removed until the bin was at least .02Mpc in width, which is around $3 \times$ the softening length. The density is spherically averaged by counting the particles within each bin and dividing by the volume of the bin. In the calculation of $\chi^{2}$, the bin is weighted by the number of particles it contains. When the fitting was particularly difficult, MPFIT would sometimes return a value of 0.0 or 1.0 for 2 at low snapshot number. This seemed to be a feature of how the value was stored within the program so we ignore these values in our analysis. Lastly, the minimum number of particles to consider an NFW fit was 50 and this was the threshold used for all other RMs as well.

(iii) Substructure mass fraction [4]:

$f_{\text {sub }}$ is the total mass inside substructures whose centers are within the virial radius of the center of the main halo divided by the mass of the main halo. This value should reasonably be between 0 and 1 but may rise above 1 if there is complex subhalo structure contained within the halo as well as a large subhalo whose center lies at the outskirts of $r_{v i r}$.

(iv) Center of mass displacement [4]:

Take the difference between the average position of particles in the halo (the center of mass) with the position of the particle at the bottom of the potential well. A low $s$ value describes a radially symmetric mass distribution.

$$
s=\frac{\left|r_{c}-r_{c m}\right|}{r_{v i r}}
$$

(v) Virial fraction [4]:

Compute $2 T /|U|$ where $T$ is the total kinetic energy of the halo particles within $r_{v i r}$ (with respect to the halo center) and $U$ the gravitational potential. This value addresses the dynamic state of the halo and how close it is to dynamic equilibrium. 


\section{Results and discussion}

This section is organized as follows: Section 3.1 deals with measurements taken to establish a connection between properties of the halo at low redshift and characterizations of the full trajectory of the halo's growth. Section 3.2 focuses on behavior of RMs around major mergers. Note also that while the Rhapsody catalog officially contains 110 halos, 106 were put through the following analyses.

Definition of Major Merger: In this project, a major merger is said to occur at timestep $t_{i}$ when $M_{i-1} / M_{i}<0.70$ where $M_{i}$ is the mass within the virial radius of the center of the halo at time $i$. This gives a distribution of most-recent MM as seen in Figure 3:

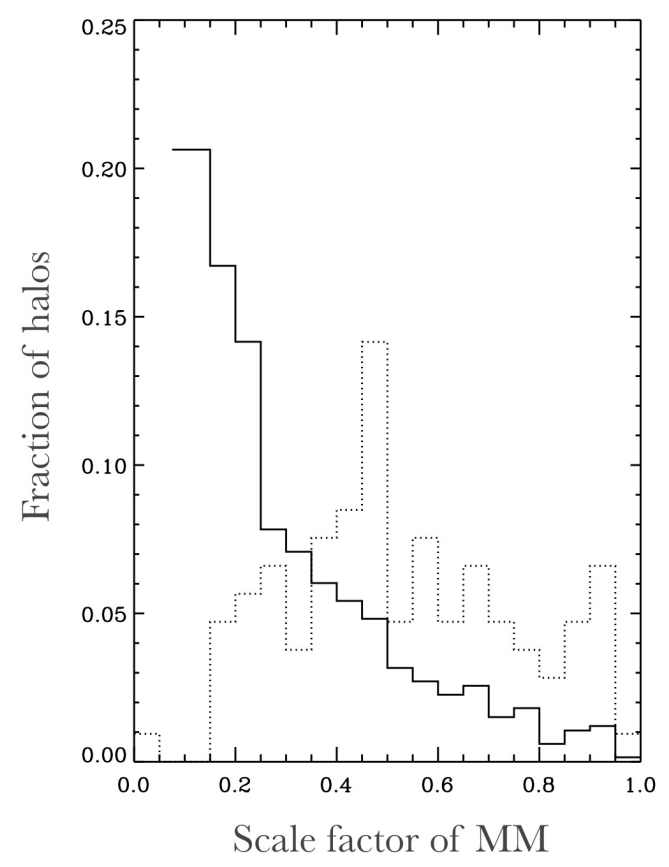

Figure 1: Fraction of all major mergers occuring within a scale factor bin (solid). The fraction of halos' most recent MM occurring within the bin is overplotted in the dotted line. 


\subsection{Relaxedness measures and halo properties}

Figure 3.1 gives an array of plots with the RMs plotted against one another with information taken at $a=1.0$ and Table 3 gives Spearman ranked-correlations. The clearest relations appear between $f_{\text {sub }}$ and $s, f_{\text {sub }}$ and $c$, and $f_{\text {sub }}$ and $s$. The more mass is in substructures, which orbit the center, the less likely it is for the distribution to be isotropic. Thus in cases of higher $f_{\text {sub }}$ and $s$ values, the mass has a wider spatial distribution, skewing the spherically averaged concentration calculation higher.

Concentration has been shown to correlate with formation time [8]. Defining when a halo has "formed" can be a bit arbitrary, though it is clear some sort of time marking when halo gained some proportion of its mass would differentiate halos. While the final mass of all 106 halos lie within a narrow mass range, their rates of accumulating mass can be quite diverse.

$a_{1 / 2}$ is defined as $\frac{1}{1+z_{1 / 2}}$ where $z_{1 / 2}$ is the redshift at which the halo first exceeds half of its final mass $\left(.5 M_{0}\right)$. Figure 3.1 shows the distribution of $a_{1 / 2}$ values for the halos.

Another way of characterizing the formation history is by fitting the full trajectory of the mass accretion history (MAH). In [8], this can be fitted by a simple power law with one parameter $\alpha$ and this model is adapted here ( $S$ is an arbitrary threshold):

$$
\frac{M(a)}{M_{0}}=e^{-\alpha z}
$$

Where $M_{0}$ is the mass of the halo at $z=0$. And the characteristic epoch of formation, denoted $a_{c}$, can be derived from:

$$
a_{c}=\frac{a_{0} \alpha}{S}=\frac{\alpha}{2}
$$

Since $a_{0}$, our intial scale factor, is 1 , and the same value of $S$ as [8] is taken. While $a_{1 / 2}$ has less scatter in its calculation, it also does not incorporate as much information of the 


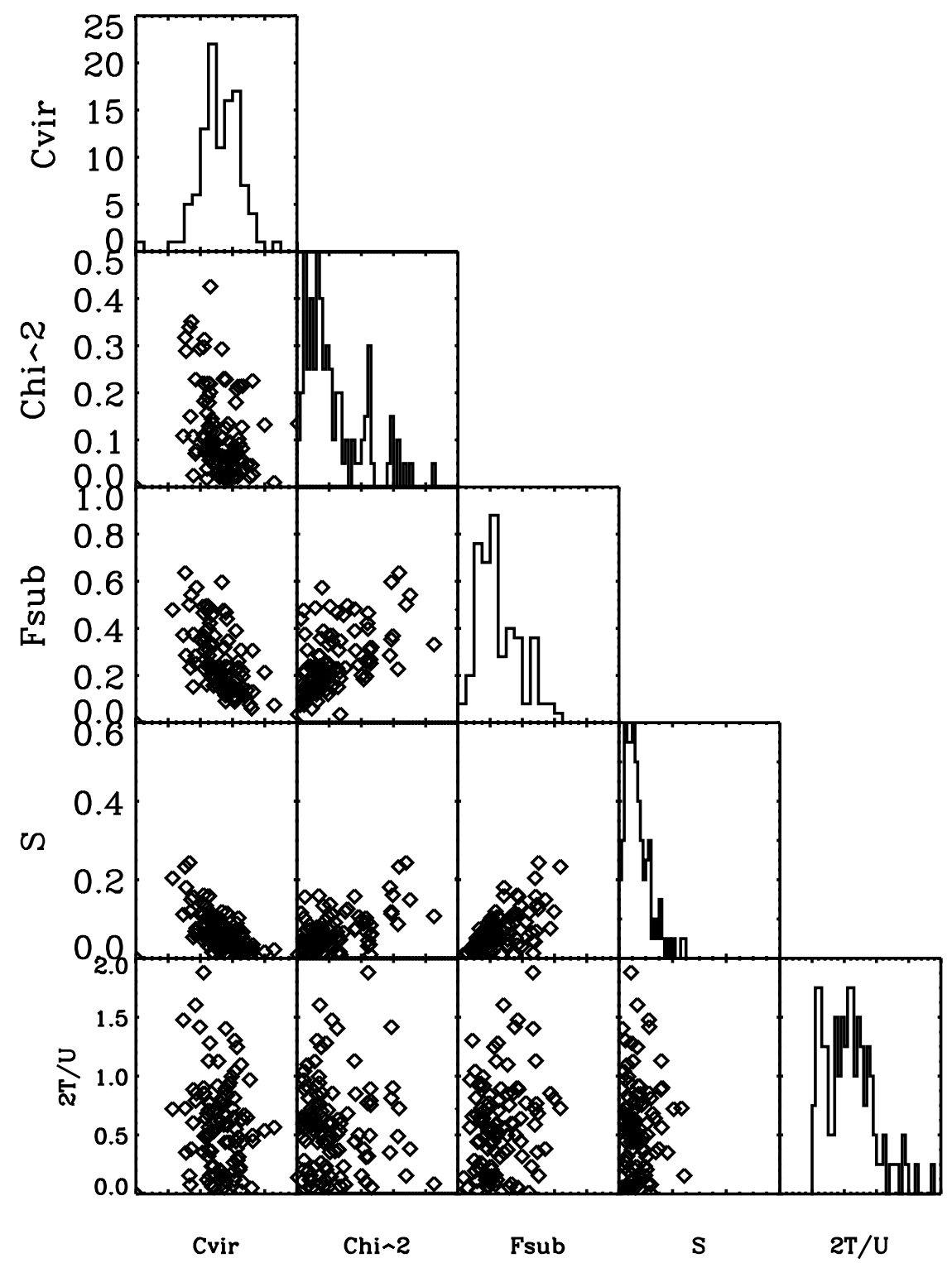

Figure 2: Off-diagonal plots are scatter plots of the relaxedness measurements at $a=1.0$. On the diagonal are histograms of the values.

MAH as $a_{c}$ does. These characterizations of the MAH are plotted against the RMs taken at $a=1.0$ in Figure 3.1 with correlation statistics in Table 7. It happens that the correlation between $a_{c}$ and last MM time is higher than that for $a_{1 / 2}$ but for both measurements we get strong anti-correlations with concentration. This agrees with the strong correlation between 
Figure 3: Table of correlation and significance values for the relationships examined in 3.1. The values are given as they appear left to right and top to bottom in 3.1

\begin{tabular}{|l|c|c|}
\hline Measure vs. Measure & R - correlation & $p$-value \\
\hline$C_{\text {vir }}$ vs. $\chi^{2}$ & -0.367836 & $9.70663 \mathrm{e}-05$ \\
\hline$C_{\text {vir }}$ vs. $f_{\text {sub }}$ & -0.587884 & $2.78713 \mathrm{e}-11$ \\
\hline$\chi^{2}$ vs. $f_{\text {sub }}$ & 0.579312 & $6.26867 \mathrm{e}-11$ \\
\hline$c_{\text {vir }}$ vs. $s$ & -0.579909 & $5.92881 \mathrm{e}-11$ \\
\hline$\chi^{2}$ vs. $s$ & 0.447961 & $1.31010 \mathrm{e}-06$ \\
\hline$f_{\text {sub }}$ vs. $s$ & 0.607516 & $3.97083 \mathrm{e}-12$ \\
\hline$c_{\text {vir }}$ vs. virial ratio & -0.117528 & 0.227964 \\
\hline$\chi^{2}$ vs. virial ratio & -0.000186086 & 1.00000 \\
\hline$f_{\text {sub }}$ vs. virial ratio & 0.189561 & 0.0505157 \\
\hline$s$ vs. virial ratio & 0.0116872 & 0.904910 \\
\hline
\end{tabular}

Figure 4: Table of correlation and significance values of relaxedness values and concentration with the time of the last major merger.

\begin{tabular}{|l|c|c|}
\hline Measure vs. Last MM & R - correlation & $p$-value \\
\hline$C_{\text {vir }}$ & -0.435212 & $2.80388 \mathrm{e}-06$ \\
\hline$f_{\text {sub }}$ & 0.437618 & $2.43454 \mathrm{e}-06$ \\
\hline$s$ & 0.278723 & 0.00364790 \\
\hline$\chi^{2}$ & 0.291659 & 0.00230319 \\
\hline virial ratio & 0.0778430 & 0.425470 \\
\hline
\end{tabular}



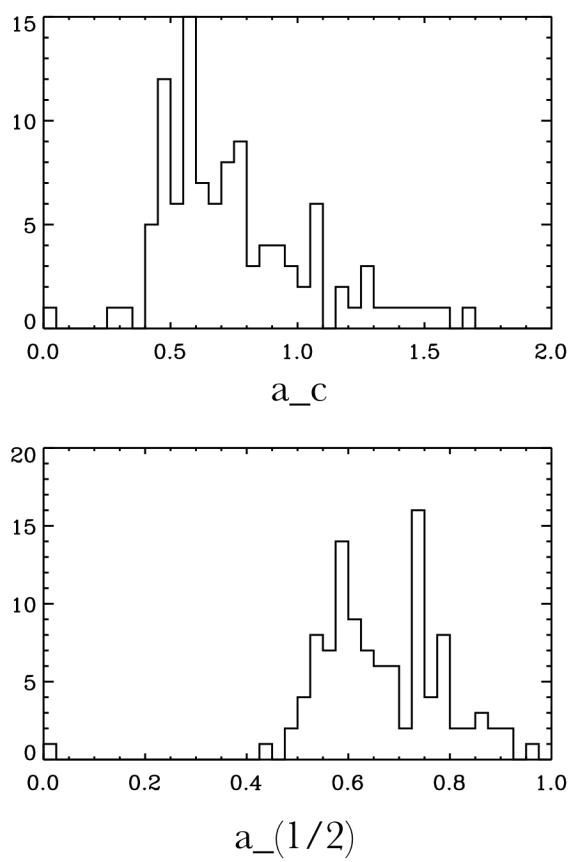

Figure 5: At left, a histogram of the $a_{c}$ (top) and $a_{1 / 2}$ (bottom) values calculated for the 106 halos considered in the sample. At right, the $a_{1 / 2}$ distribution is reproduced but with smaller bin size to show detail

$z_{1 / 2}$ and $c_{200}$ in [2]. Though in that paper, the correlation between $z_{1 / 2}$ and $c_{200}$ is stronger than that between $c_{200}$ and $f_{s u b}$. But they find both correlations to be strong. In general, among the common measurements, all of our $\left|R_{s}\right|$ values are higher than those found in [2] but the relative values between relations are indeed similar.

Among relaxedness measures, $\chi^{2}$ has the weakest correlation. Furthermore, one can still see a strong distinction in the MAHs when halos are taken from the ends of the distribution of the RMs at $a=1.0$. For the measurements of $c_{v i r}, f_{s u b}, s, \chi^{2}$, and $2 T / U$, the halos with the top and bottom $20 \%$ values (21 halos) are taken and at each timestep the median value of their mass history is plotted. The results are seen in Figure 8. How well the measure does in distinguishing the two regimes could be taken as the maximum separation between the two averages for each measurement. Incidentally, the measurement with the largest separation is concentration. At this point it is noted that the virial ratio correlates poorly to the other RMs and as seen in Figure 8 does little to separate out halos' histories. This may be due to 
the omission of a surface pressure term [5] but the measurement will be dropped from most of following discussion.

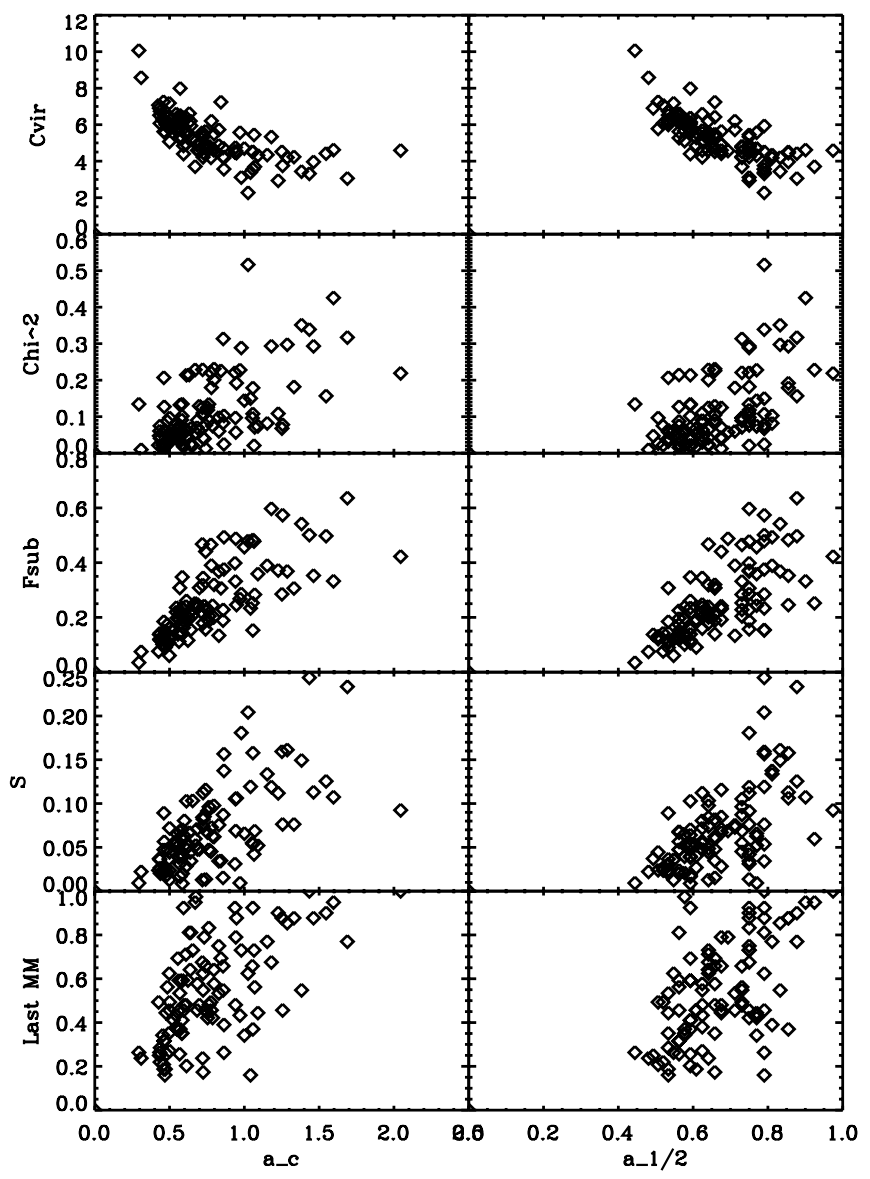

Figure 6: Different definitions of formation time are plotted against RMs at $a=1.0$ and concentration. See Table 7 for correlation values

\subsection{Relaxedness and major mergers}

How recent states of relaxed measures correlate with the full trajectory of the MAH is affected by how such measures behave around major merger events. A major merger implies an unrelaxedness but the response seen in the RMs may not be seen directly, nor solely, at the reported time of the major merger since a merger may be ongoing for several snapshots. There is also a question of where do we want to look for MMs in most of our analysis. At 
Figure 7: Table of correlation and significance values for the relationships examined in 3.1. The values are given as they appear left to right and top to bottom in 3.1

\begin{tabular}{|l|c|c|}
\hline Formation time vs. Measure & R - correlation & $p$-value \\
\hline$a_{c}$ vs. $C_{v i r}$ & -0.746015 & $2.98065 \mathrm{e}-20$ \\
\hline$a_{1 / 2}$ vs. $C_{v i r}$ & -0.743025 & $5.05537 \mathrm{e}-20$ \\
\hline$a_{c}$ vs. $\chi^{2}$ & 0.583755 & $4.13028 \mathrm{e}-11$ \\
\hline$a_{1 / 2}$ vs. $\chi^{2}$ & 0.541274 & $1.74920 \mathrm{e}-09$ \\
\hline$a_{c}$ vs. $f_{\text {sub }}$ & 0.810633 & $3.74928 \mathrm{e}-26$ \\
\hline$a_{1 / 2}$ vs. $f_{\text {sub }}$ & 0.737966 & $1.21578 \mathrm{e}-19$ \\
\hline$a_{c}$ vs. $s$ & 0.651351 & $3.04152 \mathrm{e}-14$ \\
\hline$a_{1 / 2}$ vs. $s$ & 0.621948 & $8.68429 \mathrm{e}-13$ \\
\hline$a_{c}$ vs. MM time & 0.592732 & $1.74371 \mathrm{e}-11$ \\
\hline$a_{1 / 2}$ vs. MM time & 0.509901 & $2.0259 \mathrm{e}-08$ \\
\hline
\end{tabular}
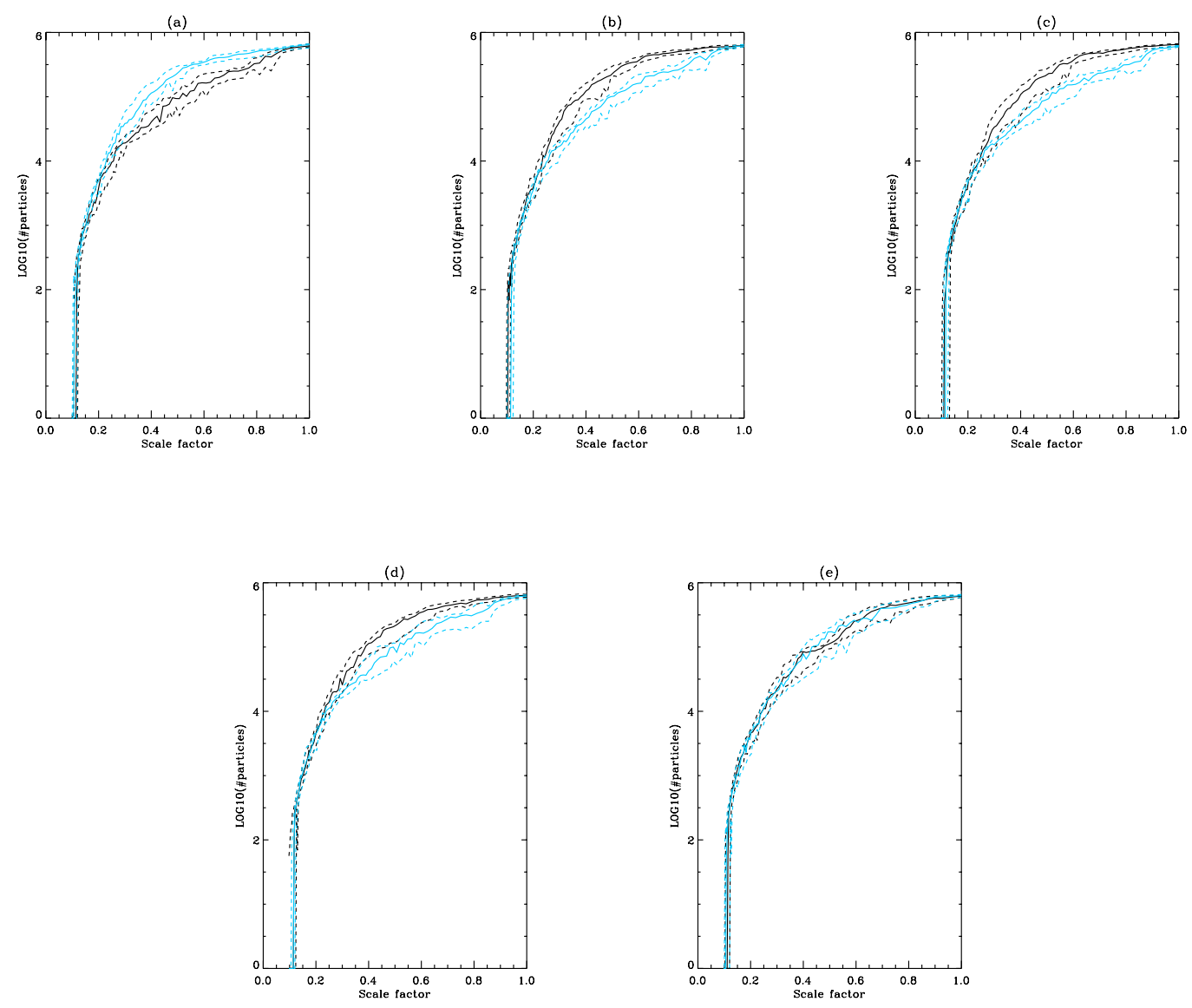

Figure 8: Solid lines show the averaged MAH for top 21 halos (black) and bottom 21 halos (blue) for 5 measures. Dashed lines show $25 \%$ and $75 \%$ values. (a) $C_{v i r}$, (b) $f_{\text {sub }}$, (c) $s$, (d) $\chi^{2}$, (e) virial ratio. 
early times, the mass may jump considerly with the addition of only 1000 particles. In Figure 3.2 is plotted the distribution of relaxed measures across all recorded snapshots on the left, and at all major mergers on the right with values of the 183 major mergers occurring after $a=.4$ overplotted. Looking at the MM at all times introduces too much noise from the measurements of relatively few particles whereas the distribution for $a>.4$ shows a larger in mean value.
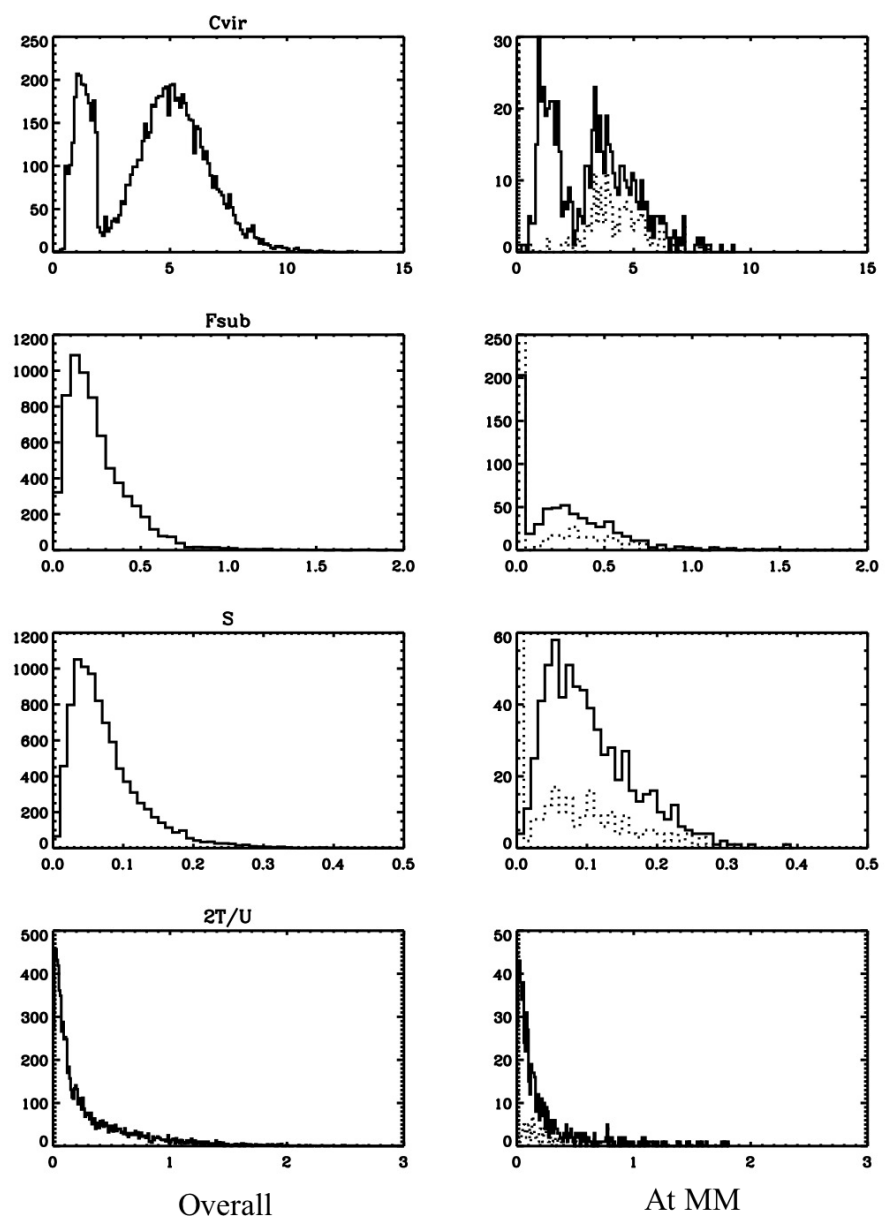

Figure 9: At left are the distributions of the RMs across all recorded snapshots and at left the distributions of values that occur at MM. In particular, the values for MMs occurring after $a=.4$ are shown in the dotted line.

Very simply, one can look at the initial response of the RMs to the major merger. Figure 10 shows the values of $f_{\text {sub }}$, and $s$ normalized to the value taken on at the MM and for halos whose last MM occurred after $a=.4$ for 3 snapshots before and 4 snapshots after 
the major merger (this resulted in 68 halos). $f_{\text {sub }}$ and $s$ where chosen since they have the strongest correlations to formation time and unlike concentration have little systematic error in their measurements. The lines are colored to reflect the jump in mass from the previous snapshot to this one, thus bluer lines indicate smaller jumps in mass and green/yellow lines more so. Since the lines are converge to 1 at time 0.0 , the radial distribution of colors on both sides of the vertical through that point give some indication of how $f_{\text {sub }}$ immediately changes around the MM for different mass jumps. Particularly for $f_{\text {sub }}$ the green lines are concentrated within $26^{\circ}$ of the vertical, and the most dramatic drops from before the MM to the MM are by lower mass jumps, for which the merger may have already mostly happened. Little besides an intuititon for the varied responses in the structure to the major merger can be deduced from Figure 3.2, so Figure 3.2 gives boxplot information for bins .5 Gyr in width. $f_{\text {sub }}$ values depart from the MM value in a much narrower range than does $s$ though there is a $60 \%$ spread in values between the upper and lower quartiles. Given that $s$ often exhibits a periodic nature when looked at across snapshots - presumably as structures cross and re-cross the halo - this seems reasonable. In this mass range it is noted that younger halos are less able to disrupt their substructures [1] so the extended presence of mass in substructures is also unsurprising. Furthermore, our definition of MM time helps enable this since it is set when these particles first enter the virial radius.

It is logical to then ask how long a halo stays "unrelaxed". Again $f_{\text {sub }}$ and $s$ are considered. To determine when a halo first becomes unrelaxed after a MM, the thresholds set by [4] were first used. These were $f_{\text {sub }}<.1$ and $s<0.07$. For $s$, this threshold still allowed us to see a significant number of halos to settle. For $f_{\text {sub }}$, only 3 out of 68 settled within the time from their MM to $a=1.0$. It took those three halos, 11,18, and 17 snapshots to get below .1. The average $f_{\text {sub }}$ value at the time of major merger was .509 among these 68 halos. So to look at more points, a threshold of .20 was selected. In this case, only 8 were not able to relax and these halos were given a "time to relax" of $\infty$. Interestingly, there seemed no evident 

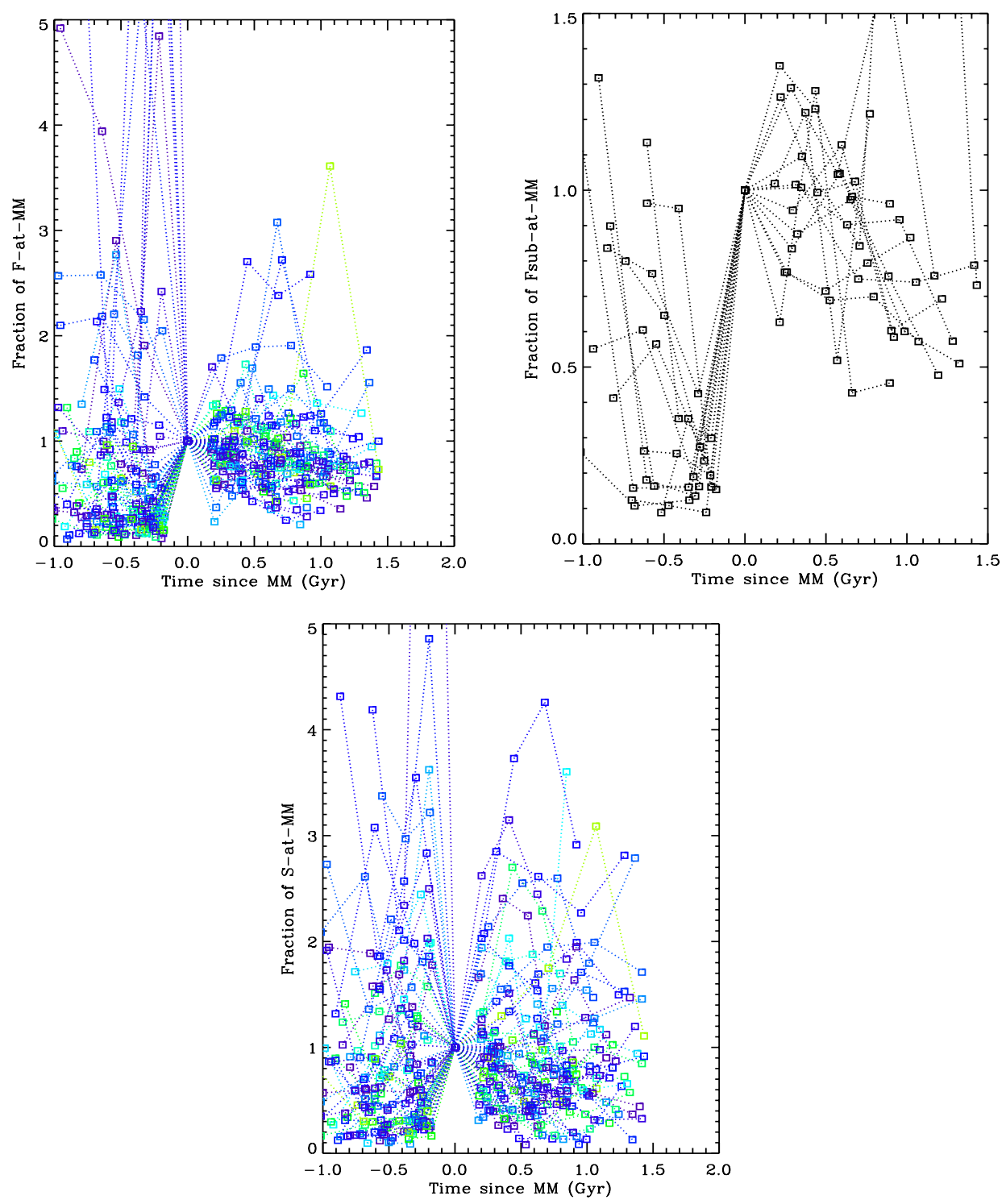

Figure 10: A stacked view of all the MMs occurring after $a=0.04$ and within 4 snapshots of $a=1.0$ where the RM value normalized to $R M\left(a_{m m}\right)$ is plotted against the time in Gyr from the major merger. A negative value implies such a time before the MM. The value at left is for $f_{\text {sub }}$ and on the bottoms. The top right isolates the halos with $\log _{10}\left(M_{f} / M_{i}\right)>.5$. The line color attaches lower values of the mass jump $\left(\log _{10}\left(M_{f} / M_{i}\right)\right)$ to the bluer end of the spectrum and higher values to the redder. 

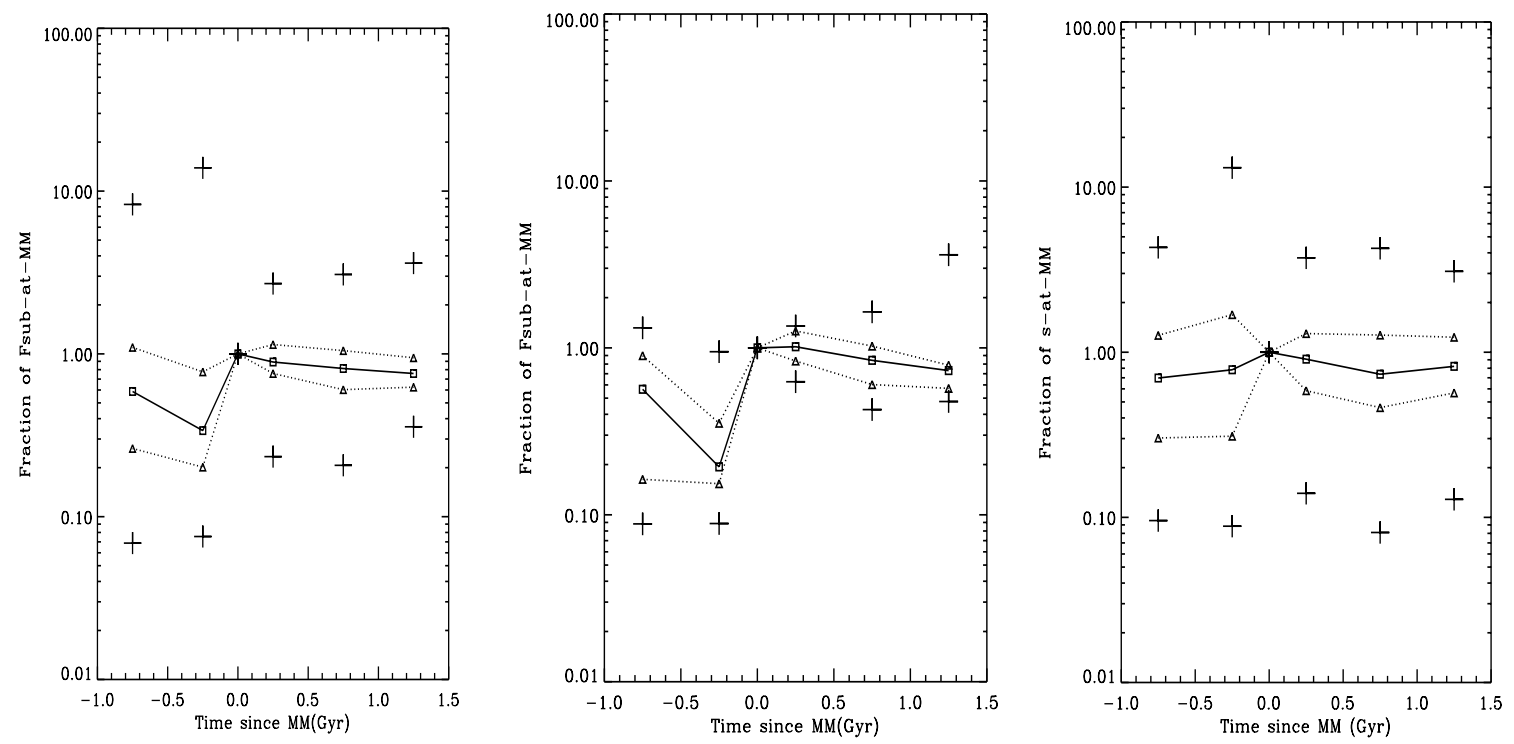

Figure 11: These plots describe the information in Figure 10. Time is binned by 0.50 Gyr. The solid line connects the medians, the dotted lines the upper and lower quartiles and the plus signs the maximums and minimums.
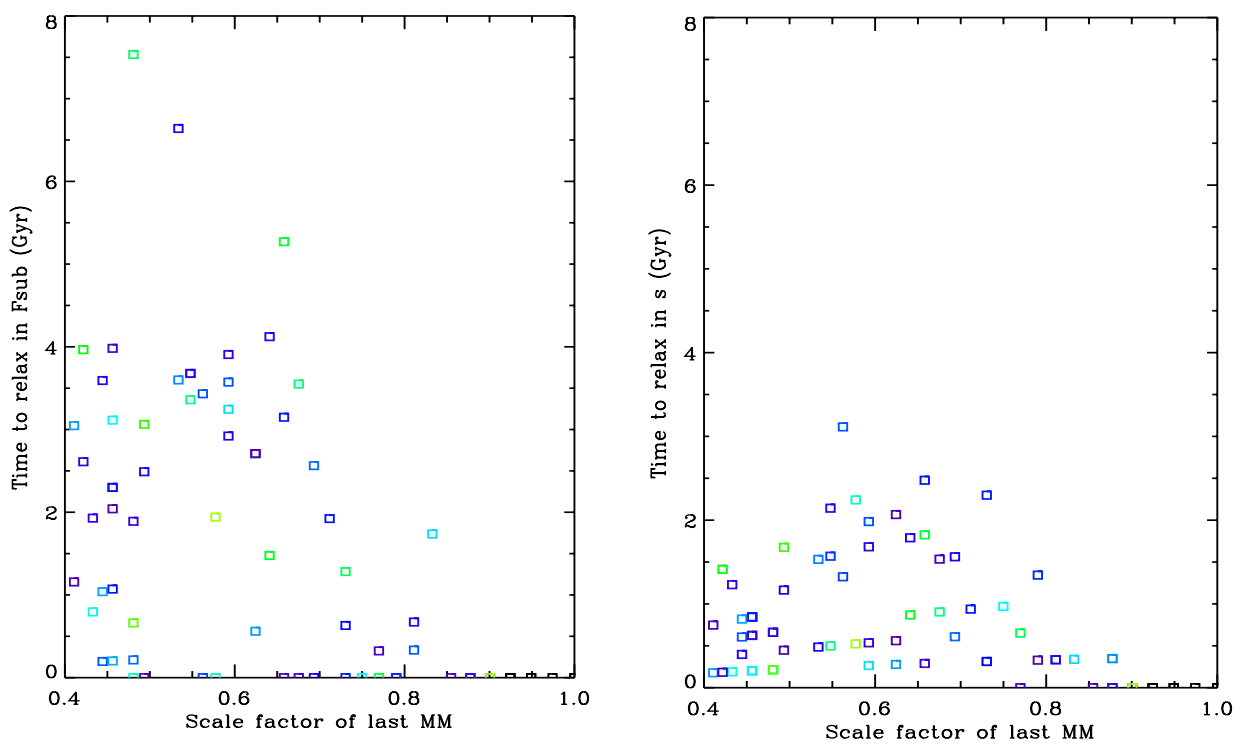

Figure 12: The scale factor of a halo's last MM and the period afterwards that it remained in an "unrelaxed" according to different RM measures. The colors correspond to the mass gained at that snapshot, lower - bluer, higher-redder.

correlation between the period of unrelaxedness and time of last MM (and again, we are considering only the most recent MM for a given halo) and to the mass ratio occurring at the MM (see Figure 12. Possible effects may come from the $f_{\text {sub }}$ curves being un-smoothed. 
Halos that we would not expect to relax as quickly maybe only dip below the threshold for a single snapshot. Something may emerge if the halos were separated by formation time or similarly by the fraction of their final mass gained at this step. This may also be a sampling issue since this analysis considered MMs in a wide time range and yet there stills seems to be evidence that by $a=1.0$, traces ostensibly left by MMs provided enough information with which correlate formation time with RMs.
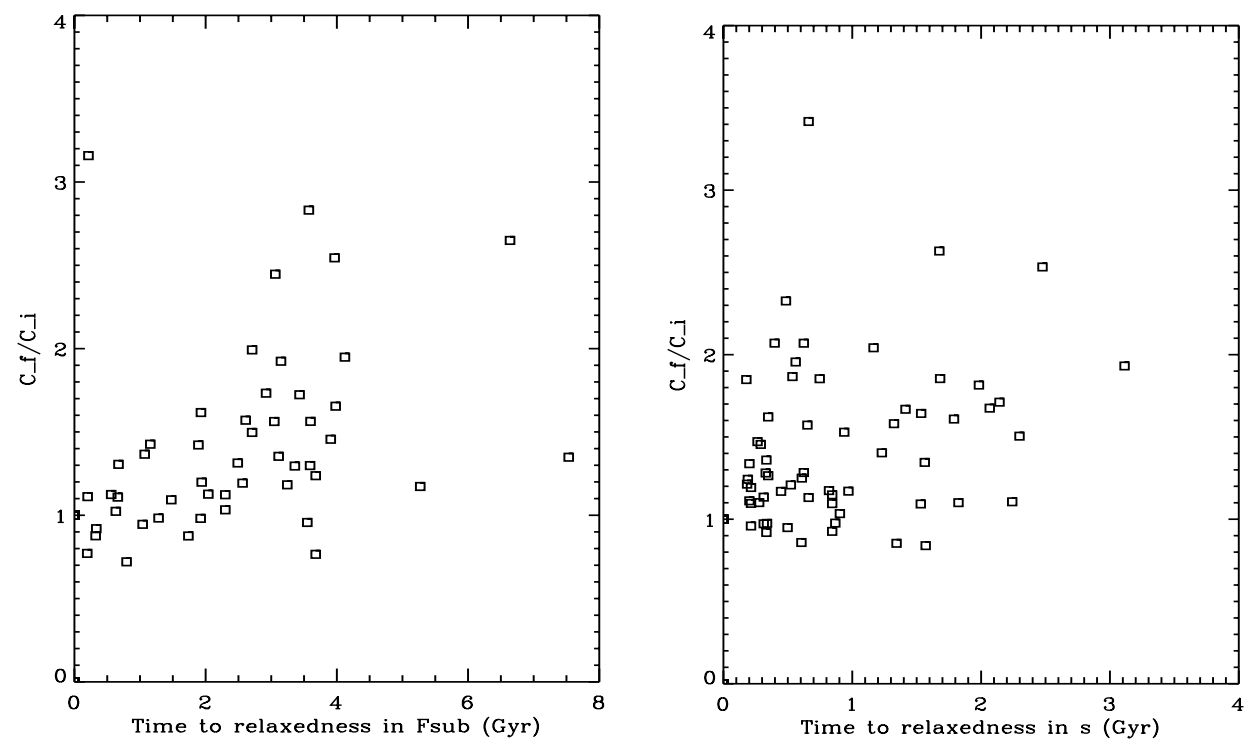

Figure 13: Relating the period of time it look for the RM to go below the relaxedness thresholds - chosen here as 0.20 for $f_{\text {sub }}$ and 0.07 for $s$ - and the change concentration underwent during this time period. The change we observe is the ratio $c_{f} / c_{i}$ where $c_{f}$ is the concentration when the RM becomes relaxed and $c_{i}$ the the concentration when the MM occurred.

An interesting relationship we do observe was with respect to how this period of unrelaxedness related to how the concentration changed over that time period. In Figure 13 we see the ratio of final to initial concentrations taken with respect to this period of unrelaxedness after a MM. The $f_{\text {sub }}$ plot at left $\left(R_{s}\right.$ value of 0.687901$)$ suggests that the shorter this period of unrelaxedness, the smaller gain in concentration. Yet Figure 12 would motivate that this happens in a way that is not completely dependent on the amount of mass gained at the time of the MM. The absence of correlation with respect to the time $s$ is unrelaxed may be 
because of the smaller timescales in comparison to $f_{\text {sub }}$. Though even when we looked at the ratio for the $f_{\text {sub }}$ time period with the time spent unrelaxed in $s$, there was little correlation.

\section{Conclusion and future work}

With sample of 106 massive halos we were able to examine relationships among relaxedness measures and see how much relaxedness measurements relate to the evolution of the halo (by way of chosen formation times). These correlations in the end, however, are directly influenced by how these relaxedness measures respond to the most evident drivers of unrelaxedness - major mergers. When we focused on $f_{\text {sub }}$ and $s$ we saw what we expected in terms of the halo remaining unrelaxed for longer periods of time with respect to $f_{\text {sub }}$. What will certainly be interesting to look further into is how the initial concentration of the halo, the mass jump at the major merger, and scale factor of the major merger perhaps influence together the time the halo remains unrelaxed. Future work may involve looking at other major mergers besides the most recent ones and incorporate other systems of analysis aside from correlation. A technique of time sequence analysis may be particularly exciting.

\section{Acknowledgements}

This research was made possible by the United States Department of Energy Summer Undergraduate Laboratory Internship (SULI). I would like to change SLAC National Accelerator Laboratory and Stanford University for hosting me this summer. I would also like to thank my project advisor Risa Wechsler and her graduate student Hao-Yi Wu for their time, guidance, and support in this project.

\section{References}

[1] Gao, L. et al, 2011, The statistics of the subhalo abundance of dark matter haloes, Monthly Notices of the Royal Astronomical Society, vol. 410, Issue 4, 2309-2314p.

[2] Jeeson-Daniel, A., Dalla Vecchia, C., Haas, Marcel R., and Schaye, J., 2011, The Correlation Structure of Dark Matter Halo Properties, Monthly Notices of the Royal Astronomical Society

[3] Navarro, J. F., Frenk, C. S., and White, S. D. M., 1996, The Structure of Cold Dark Matter Halos, Astrophysical Journal, v.462, 563p. 
[4] Neto, A. F., Gao, L., et al., 2007, The Statistics of $\Lambda$ CDM halo concentrations, Monthly Notices of the Royal Astronomical Society, vol. 381, Issue 4, 1450-1462p.

[5] Shaw, L., Weller, J., and Ostriker, J. and Bode, P., 2006, Statistics of Physical Properties of Dark Matter Clusters, The Astrophysical Journal, vol. 646, 815-833p.

[6] Steves, Bonnie A. and Maciejewski, A.J., 2001, The restless universe: applications of gravitational n-body dynamics to planetary, stellar and galactic systems, J W Arrowsmith Ltd, Bristol, 133p.

[7] Scoville, N., Aussel H. et al., 2006, Large Structure and Galaxy Evolution in COSMOS at $z<1.1$, Bulletin of the American Astronomical Society, vol. 38, 1211p.

[8] Wechsler, R., Bullock, J., Primack, J., Kravtsov, A., and Dekel, A., 2002, Concentrations of Dark Halos from their Assembly Histories, The Astrophysical Journal, vol. 568, 52-70p.

[9] $\mathrm{Wu}, \mathrm{H}$. et al, In preparation 